\title{
Bot-graphy: an original technique for plant anatomy study based on metallography
}

Ricardo Montero ${ }^{1}$, Fernando Gomez ${ }^{2}$, Lorena Setten ${ }^{3}$, Eduardo Favret ${ }^{3}$ and Darío Torres ${ }^{1}$

${ }^{1}$ Comisión Nacional de Energía Atómica - Centro Atómico Constituyentes, Villa Maipú, Buenos Aires, Argentina, ${ }^{2}$ Instituto de Tecnología Sabato - Universidad Nacional de General San Martín (UNSAM), Villa Maipú, Buenos Aires, Argentina, ${ }^{3}$ Instituto Nacional de Tecnología Agropecuaria - INTA, Hurlingham, Buenos Aires, Argentina

Plant anatomists are recurrently looking for safer and faster methods to improve the observations of plants tissues, searching for images with better resolution. Nevertheless, some plant materials are difficult to handle due to their consistency, such as those with very hard tissue or with a combination of soft and hard tissues (e. g. parenchyma and sclerenchyma). In these cases, softening techniques have been developed [1]. But they do not completely solve this problem since their success depends on the thickness of the plant organ (Argentinian anatomists, pers. commun., 2020). To find a solution to this limitation, a standard procedure of metallography is proposed, as an alternative, for the study of plant organs. The standard technique consists of mounting the specimen in a castable resin (liquid polymer film), followed by grinding the sample with abrasive papers of different granulometry and observing the sample surface with optical reflection microscopy [2]. In this work, an example is shown, the basal internodes of 2.5 to $3 \mathrm{~cm}$ in diameter of sugarcane, harvested in Famaillá, Tucumán, Argentina. A portion of fresh or fixed sugarcane stem was placed in a plastic or metallic ring. Castable resin was added and dried at room temperature (Figure 1.A). The drying time depends on the thickness of the material. Then, grinding was carried out, starting with 320-mesh abrasive paper to remove the largest excess of resin until the vegetable material began to appear on the surface. Afterwards, the sample was ground using a 600-mesh abrasive paper for periods of 30 seconds, to reduce the chances of tearing the sample. The final step was carried out with a 1000-mesh abrasive paper for 15 seconds to completely eliminate the resin from the surface of the vegetable organ. The preparation time was approximately 30 minutes. Then, the sample was observed in a reflection optical microscope (Olympus BX60M).

Usually, the internodes of sugarcane are very difficult to observe if they are prepared with the traditional techniques of anatomy due to their combination of soft and hard tissues. With the technique proposed in this paper, good quality images were obtained using brightfield and darkfield reflection microscopy (Figure 1.B - G). It was possible to identify different types of tissues and get information on each of them, e.g., size, shape and cell arrangement, and cell wall thickness, without using any type of staining technique. In addition, it permits the observation of the stem in its entire diameter.

Finally, it can be said that the standard procedure of metallography proposed could be used in plant anatomy studies when optical observations are needed. It is an economic, fast and safe technique which allows researchers to process larger pieces and to obtain images with high resolution. Despite the results, there are still things to be adjusted, such as duration of the preparation, incorporating dyes if necessary, and its use in other types of organs (leaves, roots, flowers, etc.), fruits and seeds.

Authors acknowledge to Dr. Alberto Acevedo of Instituto de Suelos (INTA - Argentina) for the sugarcane's samples. 


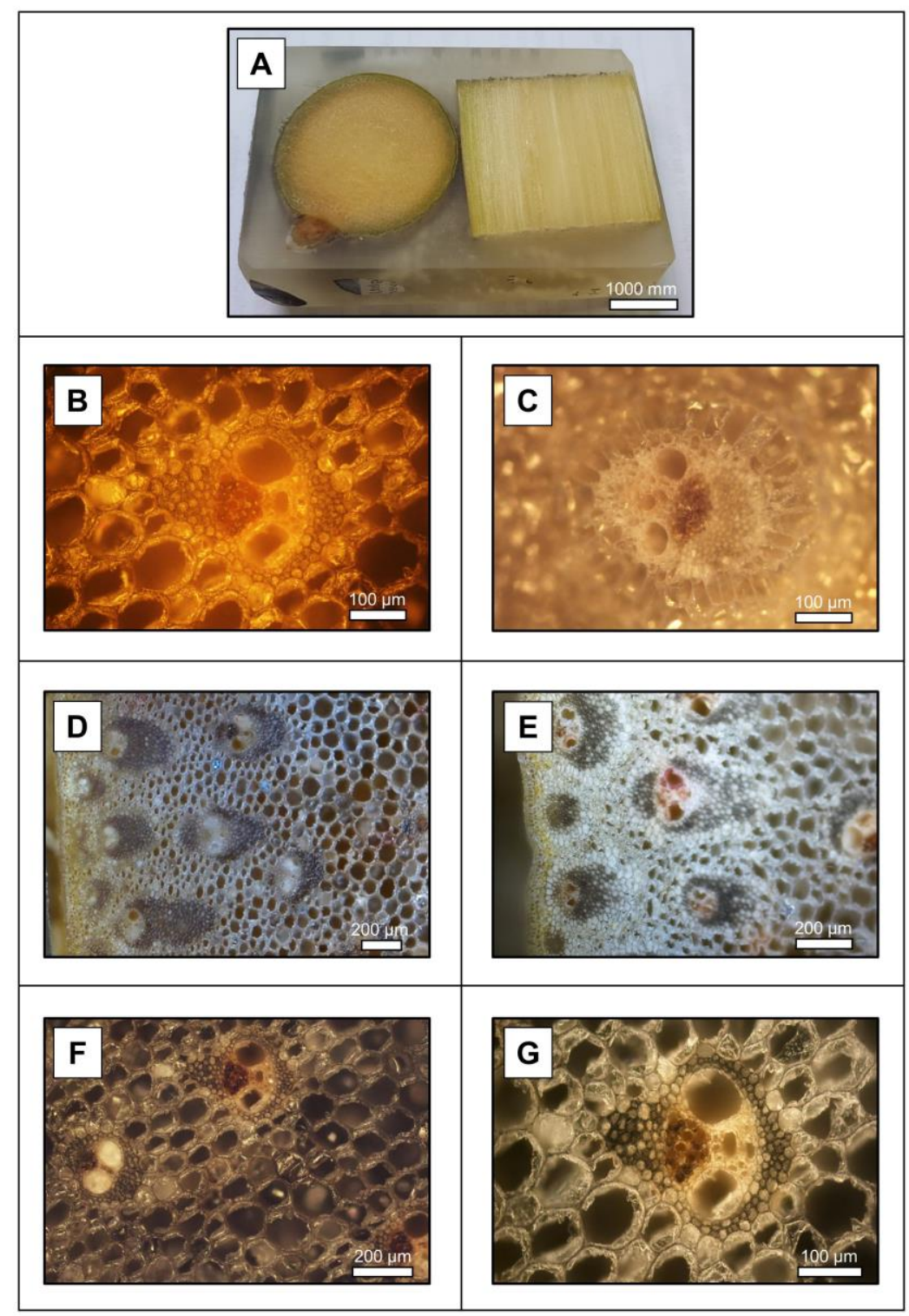

Figure 1. Basal internodes of sugarcane: A- Longitudinal and cross sections mounted in castable resin. B-C: Cross section images of vascular bundles (Brightfield reflection microscopy). D-G: Cross section images (Darkfield reflection microscopy). D-E: Cortex, F-G: Vascular bundles.

References

[1] D’Ambrogio de Argüeso, A. (1986).Manual de Técnicas en Histología Vegetal. Buenos Aires: Hemisferio Sur. 83 pp.

[2] Vander Voort, G. (1984). Metallography, Principles and Practice. McGraw-Hill, Series in Materials Science and Engineering. 752 pp. 